\title{
Real Time Deconvolution of In-Vivo Ultrasound Images
}

\author{
Jensen, Jørgen Arendt
}

Published in:

Proceedings of IEEE International Ultrasonics Symposium

Link to article, DOI:

10.1109/ULTSYM.2013.0008

Publication date:

2013

Document Version

Early version, also known as pre-print

Link back to DTU Orbit

Citation (APA):

Jensen, J. A. (2013). Real Time Deconvolution of In-Vivo Ultrasound Images. In Proceedings of IEEE International Ultrasonics Symposium (pp. 29-32). IEEE. https://doi.org/10.1109/ULTSYM.2013.0008

\section{General rights}

Copyright and moral rights for the publications made accessible in the public portal are retained by the authors and/or other copyright owners and it is a condition of accessing publications that users recognise and abide by the legal requirements associated with these rights.

- Users may download and print one copy of any publication from the public portal for the purpose of private study or research.

- You may not further distribute the material or use it for any profit-making activity or commercial gain

- You may freely distribute the URL identifying the publication in the public portal

If you believe that this document breaches copyright please contact us providing details, and we will remove access to the work immediately and investigate your claim. 
Paper presented at the IEEE International Ultrasonics Symposium, Prague, Czech Republic, 2013:

\section{Real Time Deconvolution of In-Vivo Ultrasound Images}

Jørgen Arendt Jensen

Center for Fast Ultrasound Imaging,

Biomedical Engineering group, Department of Electrical Engineering, Bldg. 349,

Technical University of Denmark, DK-2800 Kgs. Lyngby, Denmark

To be published in Proceedings of IEEE International Ultrasonics Symposium, Prague, Czech Republic, 2013. 


\title{
Real Time Deconvolution of In-Vivo Ultrasound Images
}

\author{
Jørgen Arendt Jensen \\ Center for Fast Ultrasound Imaging, Department of Electrical Engineering, \\ Technical University of Denmark, DK-2800 Lyngby, Denmark
}

\begin{abstract}
The axial resolution in medical ultrasound is directly linked to the emitted ultrasound frequency, which, due to tissue attenuation, is selected based on the depth of scanning. The resolution is determined by the transducers impulse response, which limits the attainable resolution to be between one and two wavelengths. This can be improved by deconvolution, which increase the bandwidth and equalizes the phase to increase resolution under the constraint of the electronic noise in the received signal. A fixed interval Kalman filter based deconvolution routine written in $\mathrm{C}$ is employed. It uses a state based model for the ultrasound pulse and can include a depth varying pulse and spatially varying signal-to-noise ration. An autoregressive moving average (ARMA) model of orders 8 and 9 is used for the pulse, and the ARMA parameters are determined as a function of depth using a minimum variance algorithm using averaging over several RF lines. In vivo data from a $3 \mathrm{MHz}$ mechanically rotating probe is used and the received signal is sampled at $20 \mathrm{MHz}$ and 12 bits. In-vivo data acquired from a 16th week old fetus is used along with a scan from the liver and right kidney of a 27 years old male. The axial resolution has been determined from the in-vivo liver image using the auto-covariance function. From the envelope of the estimated pulse the axial resolution at Full-Width-Half-Max is $0.581 \mathrm{~mm}$ corresponding to $1.13 \lambda$ at $3 \mathrm{MHz}$. The algorithm increases the resolution to $0.116 \mathrm{~mm}$ or $0.227 \lambda$ corresponding to a factor of 5.1. The basic pulse can be estimated in roughly 0.176 seconds on a single CPU core on an Intel i5 CPU running at 1.8 GHz. An in-vivo image consisting of 100 lines of 1600 samples can be processed in roughly 0.1 seconds making it possible to perform real-time deconvolution on ultrasound data by using dual or quad core CPUs for frame-rates of 20-40 Hz.
\end{abstract}

\section{Deconvolution}

Linear ultrasound imaging can accurately be described as a convolution between the spatial scattering map and the ultrasound field [1]. This convolution model basically consists of a one-dimensional pulse convolved with the spatial impulse responses to give the full three-dimensional field. The one-dimensional pulse essentially determines the axial resolution of the image and depends on both the transducer used and the patient. Many authors have suggested to use various deconvolution algorithms to enhance the resolution of the images. Several have been based on the Wiener filter [2], which is most easily defined in the frequency domain. It is easy to implement and fast, but it neglects the depth dependence of the one-dimensional pulse that changes as a function of depth due to the dispersive attenuation in the human body. Also the signal-to-noise ratio varies throughout the images. This stems from both the increase in noise due to the time-gain compensation amplifier as well as the spatial variation of the scattering. This varies from strong reflections from boundaries, through intermediate scattering in the liver to the weak scattering from cysts and blood. Keeping the covariance ratio between the noise power and the reflection power throughout the image will give noise in weak scattering regions and less than ideal resolution enhancements in high scattering regions. A good deconvolution algorithm should, thus, take these spatial variations into account.

Mendel [3] has developed a fixed interval Kalman filter [4] based algorithm for deconvolution of seismic signals. This algorithm is ideally suited for clinical ultrasound deconvolution as both the covariance ratio and the pulse can change from sample to sample. Furthermore it uses efficient parametric models for the pulse. It has been used in initial clinical trials on ultrasound data by Jensen et al. [5]. The purpose of this paper is to show the obtained clinical performance and demonstrate that a $\mathrm{C}$ library implementation of the method can be made to run in real-time on todays high-end multi-core CPUs. The paper introduces the various steps in the algorithm for pulse estimation, covariance estimation and deconvolution in Sections II. Validation results are shown in Sections III and IV for simulated data. Finally the performance for in-vivo data is given in Section V.

\section{Deconvolution Algorithm}

The complete deconvolution algorithm consists of three parts: pulse estimation, covariance estimation, and the Kalman based deconvolution algorithm. The first part estimates the one-dimensional pulse using a single input, single output ARMA (Auto Regressive Moving Average) model:

$$
\begin{array}{r}
\left(1+a_{1} z^{-1}+a_{2} z^{-2} \cdots+a_{n} z^{-n}\right) y(k)= \\
\left(1+c_{1} z^{-1}+\cdots+c_{n} z^{-(n-1)}\right) e(k)
\end{array}
$$

where $y(k)$ is the measured signal and $e(k)$ is the reflection signal. The parameters $a, c$ are estimated using a number of $\mathrm{RF}$ lines combined. The algorithm is described in detail in [6].

The covariance estimation is performed by finding the covariance of the RF data in a region and then dividing by the lag zero autocorrelation value of the estimated pulse. This relies on the assumption that

$$
R_{y}(\tau, \vec{r})=R_{e}(\tau, \vec{r}) * R_{p}(\tau, \vec{r})=P_{e}(\vec{r}) R_{p}(\tau, \vec{r})
$$




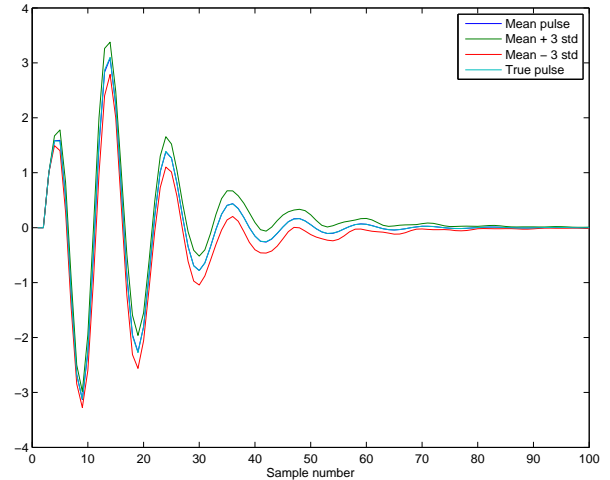

Fig. 1. Mean of the estimated pulse \pm three standard devotion. The true pulse is also shown.

where $R_{x}$ is the autocorrelation of signal $x$, and $\vec{r}$ is the spatial location for the estimation. It is assumed that the reflection sequence is white, zero mean Gaussian.

The parameters are then used in the deconvolution algorithm described by Mendel [3], [7] and used by Jensen et al. [5], where it is described in detail.

\section{Pulse estimation VAlidation}

The pulse estimation algorithm is validated by using a synthetic example with known parameters and then calculating a number of estimates to evaluate the accuracy.

The $\operatorname{ARMA}(6,6)^{1}$ model used is given by:

$$
\begin{aligned}
\operatorname{ar}(q)= & 1.0-2.3249 q^{-1}+1.590 q^{-2}+1.0265 q^{-3} \\
& -2.2165 q^{-4}+1.3192 q^{-5}-0.2665 q^{-6} \\
\operatorname{ma}(q)= & 1.0-0.7478 q^{-1}-0.5027 q^{-2}+0.4022 q^{-3} \\
& -0.5254 q^{-4}+0.3912 q^{-5}+0.0747 q^{-6}
\end{aligned}
$$

This model is convolved with a Gaussian, random signal with unit variance and the model orders and signals are then fed to the pulse estimation algorithm. The input signal has 1000 lines and 10 independent signals that are all used in the estimation. The experiment is repeated 100 times. The mean estimated waveform \pm three standard deviations are shown in Fig. 1 on top of the true impulse response. It can be seen that the estimation is unbiased and that a very low standard deviation is attained.

All 100 experiments took 17.6 seconds to conduct under Matlab using a single core on an Intel i5 CPU running on a portable PC with a clock frequency of $2.4 \mathrm{GHz}$. This also includes generating the synthetic data. A single pulse estimation can, thus, be conducted in less then 0.176 seconds for a single CPU corresponding to more than 5 frames per second. Often the view is not changed so often, and the pulse only needs to be estimated for a change in view. Therefore this should be a sufficient frame rate for real time imaging.

\footnotetext{
${ }^{1}$ An ARMA $(4,6)$ model indicates that the order of the AR part is 4 and the order of the MA part is 6 .
}
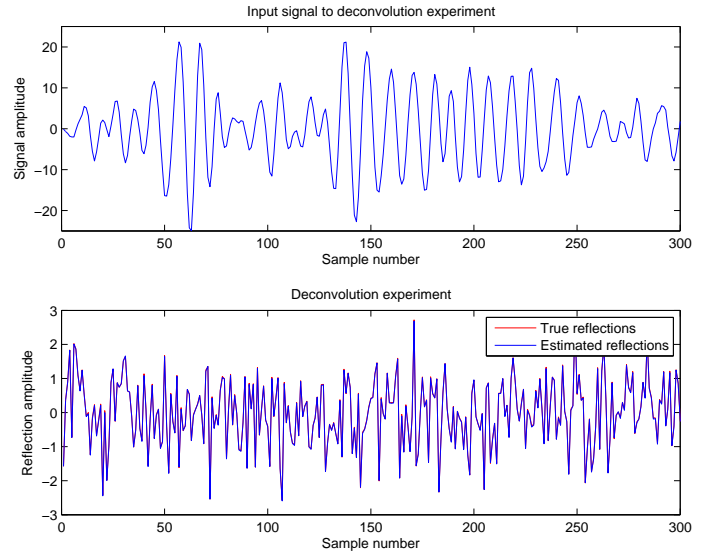

Fig. 2. Estimated reflection signal (blue line) and true reflection sequence (red line).

\section{DECONVOLUtion CODE VALIDATION}

The deconvolution algorithm is validated using synthetic data and the $\operatorname{ARMA}(6,6)$ model mentioned in the previous Section. A synthetic signal consisting of 1600 samples and 100 lines is made. For $\lambda / 4$ sampling this corresponds to a penetration depth of $400 \lambda$, which is roughly around the limit for a traditional B-mode image. No noise is added to the signal in the first example.

The routine estimates the ARMA model for the pulse using all the data, and the covariance map for the image is estimated from the parameters along with the reflection signal. The result for the first line and the first 300 samples in shown in Fig. 2 , where the blue line is the estimated reflection signal and the true reflection sequence is the red line. There is nearly a complete overlap, so the magnitude is correctly estimated without bias along with the correct placement and amplitude of the reflections.

The experiment has been conducted 100 times with a new set of $1600 \times 100$ samples and this took 10.2 seconds to execute the deconvolution part on a single core on an Intel i5 CPU running on a portable $\mathrm{PC}$ with a clock frequency of $2.4 \mathrm{GHz}$. This gives an execution time of $102 \mathrm{~ms} / \mathrm{image}$ and it is, thus, possible to make roughly 10 frame per second on a standard CPU. A four core CPU should be able to process around 40 frames/s giving real time deconvolved Bmode imaging with the $\mathrm{C}$ code split to the four cores.

The next example shows the influence of noise on the deconvolved response. Random Gaussian noise has been added to the image for different ratios with the same amount of data as in the previous example. The pulse has then been estimated using all the data and then used in the deconvolution algorithm. The power of the noise is input to the routine and it estimates the covariance of the reflection sequence before performing the deconvolution.

The power of the difference between the true reflection signal and the estimated reflections as function of signal-tonoise ratio is shown in Fig. 3. A gradual improvement is seen with the increase in signal-to-noise ratio until there essentially is no difference between the two signals at roughly 50-60 dB. 


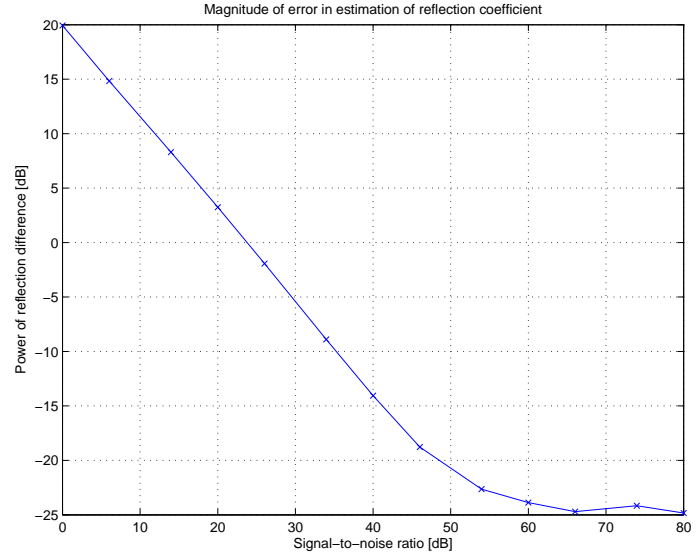

Fig. 3. Error power in estimated reflection signal compared to true reflection sequence for different signal-to-noise ratios.

The results are highly accurate for large signal-to-noise ratios whereas a ratio below $30 \mathrm{~dB}$ gives a worse result. Part of the reason for the degraded result is that the basic pulse also is poorly estimated and the amplitude is significantly lower than for the actual pulse. This gives a higher estimate of the reflection power and the estimated signal therefore has a larger amplitude than the reference signal. But as long as the SNR is around $40 \mathrm{~dB}$ or higher a very high resolution is attained. It should here be noted that a true pulse is found here, and in a real example some approximations to the actual pulse is made due to the employment of an ARMA model.

The last example uses clinical data acquired with a $3 \mathrm{MHz}$ concave round transducer obtained from a fetus in the 19th week. The data was sampled at $20 \mathrm{MHz}$ and no filtration was performed on it. The image consists of 130 lines with 0.88 degrees between lines acquired in the counter clockwise direction. Data from sample 401 is used and 2.700 samples are used both in the pulse estimation and in the deconvolution. An $\operatorname{ARMA}(9,8)$ model is used and a fixed model is estimated for all depths. The covariance of the noise has been measured in a water bath without any reflectors in and is input as a fixed value into the model. Another possible way to measure the noise covariance is to take a stable phantom, measure e.q. 10 images and subtract the mean of all measurements from the individual ones. The residual will then be the noise, which can be inserted into the deconvolution routine. The original image is shown in Fig. 5 with a dynamic range of $50 \mathrm{~dB}$. A Hilbert transform is used to find the envelope of the data.

After pulse estimation the covariance of the reflection sequence is found. The result of this is shown in Fig. 4. It can be seen how the covariance varies over the image and this is taken into account in the deconvolution routine. A large signal-to-noise ratio will give a very sharp image and for a low signal-to-noise ratio a more modest enhancement is made.

This can also be seen in the deconvolved image in Fig. 6, where a much sharper image is attained. At the same time the displayed signal is not getting poor in the black areas for the amniotic fluid, where the signal-to-noise ratio is low.

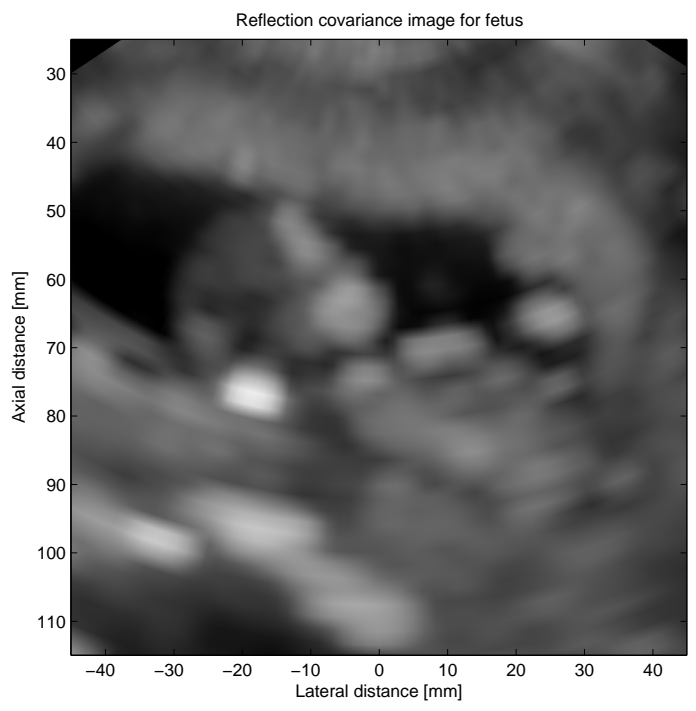

Fig. 4. Estimated covariance map of the reflection strength.

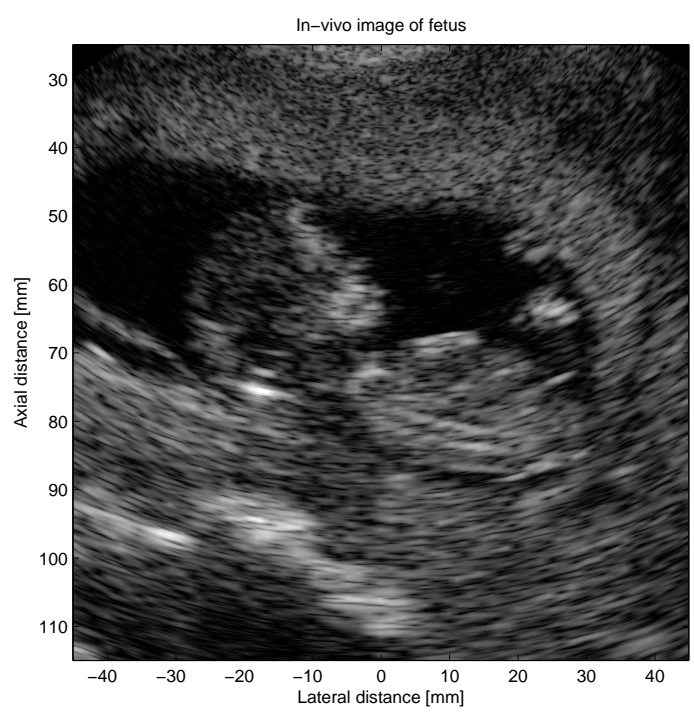

Fig. 5. Original image of fetus in the 19th week.

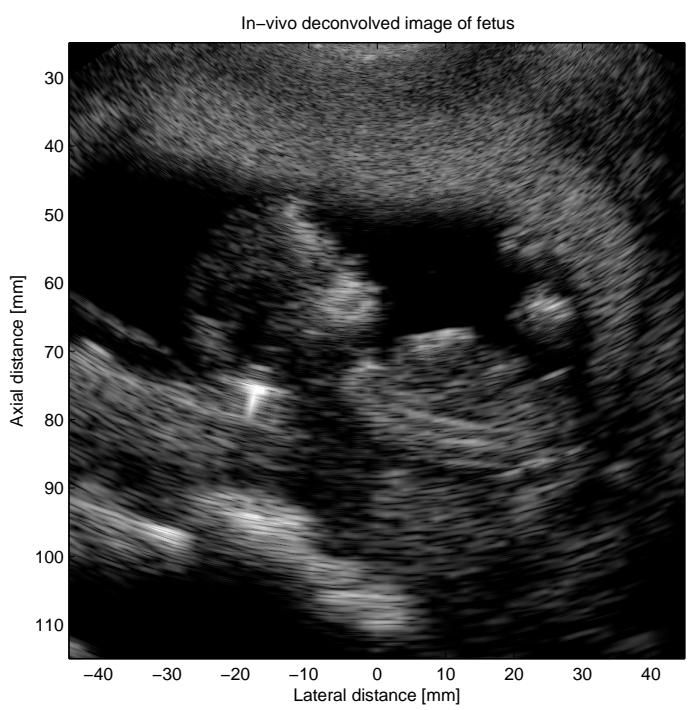

Fig. 6. Resulting deconvolved image of the fetus. 


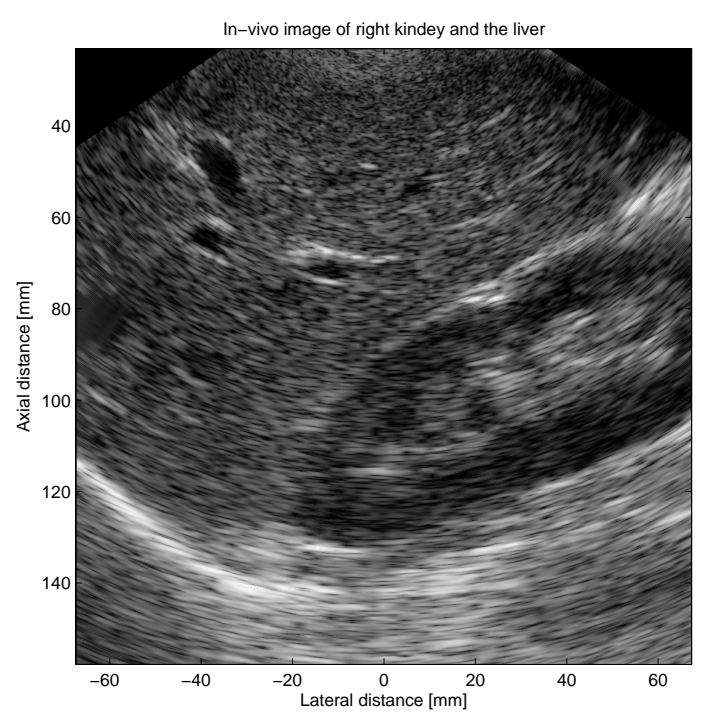

Fig. 7. Original image of the liver and the right kidney.

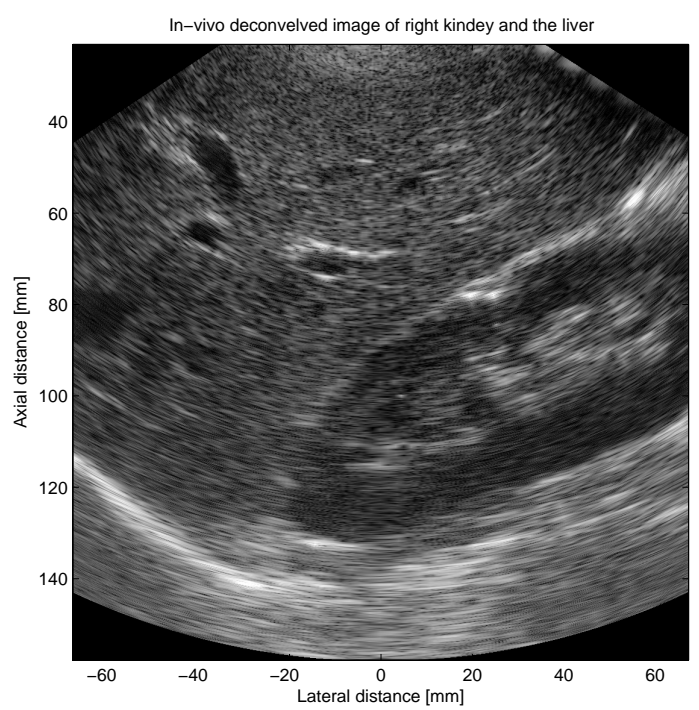

Fig. 8. Deconvolved image of the liver and the right kidney.

\section{RESOLUTION MEASURES}

The resolution obtained in the deconvolved images can be determined from the auto-covariance function of the resulting signal as

$$
R_{c}(\tau)=\sum_{i=1}^{M} \sum_{k=1}^{N}(z(k, i)-\bar{z}(k, i))(z(k+\tau, i)-\bar{z}(k, i))
$$

where $z(k, i)$ is either the RF signal or the corresponding deconvolved signal for the $i$ 'th line in the image at sample $k, N$ is the number of samples and $M$ is the number of lines. $\bar{z}(k, i)$ is the mean value of the signal. $R_{c}(\tau)$ is found from a region in the image and averaged over it and reveals the correlation length of the data.

The method has been tested on an in-vivo image of a liver and the right kidney as shown in Fig. 7 with a dynamic range of $50 \mathrm{~dB}$.

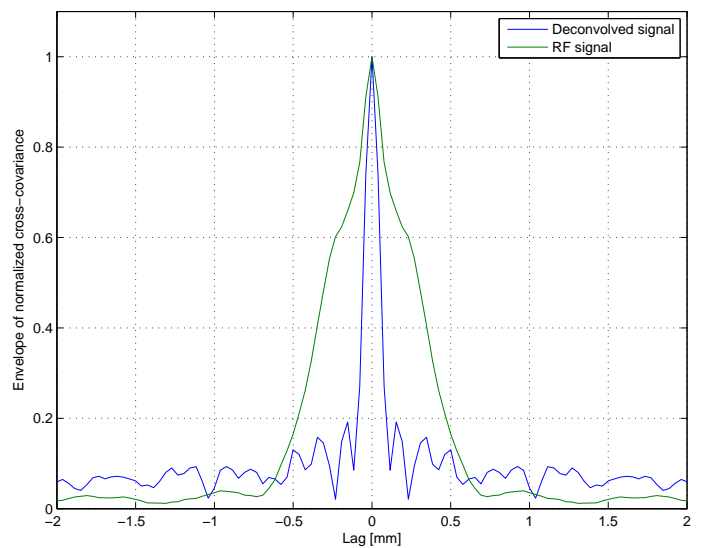

Fig. 9. Estimated envelope of the auto-covariance function for both the RF signal and the resulting deconvolved signal.

The pulse estimation is performed in the upper part of the liver, where a homogeneous speckle pattern is found. The region consists of the center 70 lines in the image each containing 1000 samples. From the envelope of the estimated pulse the axial resolution at Full-Width-Half-Max (FWHM) is $0.581 \mathrm{~mm}$ corresponding to $1.13 \lambda$ for a center frequency of $3 \mathrm{MHz}$. This resolution is typical for a high resolution clinical system. The same figure of merit can be calculated from the envelope of the auto-covariance function of both the input and deconvolved signals. These are shown in Fig. 9. The FWHM of $R_{c}(\tau)$ for the RF signal is $0.595 \mathrm{~mm}$ corresponding to $1.16 \lambda$. For the deconvolved data the FWHM is $0.1164 \mathrm{~mm}$ corresponding to $0.227 \lambda$. The increase in resolution in this clinical example is, thus, a factor of 5.1.

\section{CONCLUSION}

It has been demonstrated that clinical images can be deconvolved and the resolution increased by a factor of 5 . Depending on the signal-to-noise ratio and the transducer used a typical increase is between 2 and 5. The deconvolution can be performed in around 0.1 to $0.5 \mathrm{~s}$ depending on the size of the image. Employing modern multi-core CPUs should, thus, make it possible to implement real-time deconvolution of invivo ultrasound images on a modern PC.

\section{REFERENCES}

[1] J. A. Jensen, "A model for the propagation and scattering of ultrasound in tissue," J. Acoust. Soc. Am., vol. 89, pp. 182-191, 1991.

[2] N. Wiener, Extrapolation, interpolation and smoothing of stationary time series, with engineering applications. New York: Wiley \& Sons, Inc., 1949.

[3] J. M. Mendel, Optimal seismic deconvolution, An estimation-based approach. Academic Press, 1983.

[4] R.E.Kalman, "On the general theory of control systems," in Proc.First.IFAC Congress, Moscow, vol. 1, 1960, pp. 481-492.

[5] J. A. Jensen, J. Mathorne, T. Gravesen, and B. Stage, "Deconvolution of in-vivo ultrasound B-mode images," Ultrason. Imaging, vol. 15, pp. 122-133, 1993.

[6] J. A. Jensen, "Estimation of in-vivo pulses in medical ultrasound," Ultrason. Imaging, vol. 16, pp. 190-203, 1994.

[7] J.M.Mendel and J.Kormylo, "New fast optimal white-noise estimators for deconvolution," IEEE Trans. Geo. Elec., vol. GE-15, pp. 32-41, 1977. 